\title{
ON TOEPLITZ OPERATORS WHICH ARE CONTRACTIONS
}

\author{
ROBERT GOOR
}

Abstract. We prove that a Toeplitz contraction $T_{\phi}$ is completely nonunitary if $\phi$ is not a constant. As an application, it is noted that for such $T_{\phi}$, a functional calculus can be defined for all functions $u$ in $H^{\infty}$ of the unit disk.

For $1 \leqq p \leqq \infty$, we denote by $L^{p}$ the usual class of Lebesgue measurable functions on the unit circle $\gamma$ of the complex plane. We write $H^{p}$ for the closed subspace of $L^{p}$ of functions whose Fourier coefficients vanish on the negative integers. We denote by $P$ the orthogonal projection of $L^{2}$ onto $H^{2}$ and by $B\left(H^{2}\right)$ the space of bounded operators on $H^{2}$. For $\phi \in L^{\infty}$, we consider the Toeplitz operator $T_{\phi} \in B\left(H^{2}\right)$ defined by $T_{\phi} f=P(\phi f)$ for $f \in H^{2}$. Following Sz.-Nagy and Foiaş [2], we say a contraction $T_{\phi}$, $\left\|T_{\phi}\right\| \leqq 1$, is completely nonunitary (c.n.u.) if $T_{\phi}$ has no nontrivial reducing subspaces restricted to which $T_{\phi}$ is unitary. We will use the fact that $T_{\phi}^{*}=T_{\bar{\phi}}[1, \mathrm{p} .137]$.

THEOREM. If $\phi \in L^{\infty},\|\phi\|_{\infty} \leqq 1$ and $\phi$ is not a constant (almost everywhere), then $T_{\phi}$ is c.n.u.

Proof. Suppose $T_{\phi}$ is not c.n.u. We will show that $\phi$ is constant. Let $S$ be a nontrivial reducing subspace for $T_{\phi}$ on which $T_{\phi}$ is unitary. We may write $S=\left\{f \in H^{2}:\left\|T_{\phi}^{n} f\right\|_{2}=\|f\|_{2}=\left\|T_{\phi}^{n} f\right\|_{2}\right.$ for $\left.n=1,2, \cdots\right\}$. Now, for $f \in S,\|f\|_{2}=\left\|T_{\phi} f\right\|_{2}=\|P \phi f\|_{2} \leqq\|\phi f\|_{2} \leqq\|f\|_{2}$ and the resulting equality gives $\phi f \in H^{2}$. Similarly, $\phi^{n} f$ and $\bar{\phi}^{n} f \in H^{2}$ for $n \geqq 0$ and $f \in S$. We may apply the F. and $\mathrm{M}$. Riesz theorem [1, p. 82] to the equality $\|\phi f\|_{2}=\|f\|_{2}$ for a nonzero $f \in S$ to conclude that $|\phi|=1$ almost everywhere on $\gamma$. Thus, we write $S=\left\{f \in H^{2}: \phi^{n} f, \bar{\phi}^{n} f \in H^{2}\right.$ for $\left.n \geqq 0\right\}$.

Let $M_{z}$ be the operator of multiplication by the coordinate function $z$. Then $\phi^{n} f \in H^{2}$ implies $z \phi^{n} f=\phi^{n} z f \in H^{2}$ and similarly for $\phi^{n} z f$, i.e. $M_{z} S \subset S$. By Beurling's theorem [1, p. 79], there is a function $\psi \in H^{\infty}$, $|\psi|=1$ almost everywhere, such that $S=\psi H^{2}$. Since $1 \in H^{2}, \psi$ is in $S$. Note that $\phi \psi=\psi f$ for some $f \in H^{2}$ (since $\phi \psi \in S=\psi H^{2}$ ). Hence for

Received by the editors November $15,1971$.

AMS 1970 subject classifications. Primary 47B35, 47A20, 47A60; Secondary 46J15.

Key words and phrases. Toeplitz operator, contraction, completely nonunitary, reducing subspace, Beurling theorem, F. and $\mathrm{M}$. Riesz theorem.

(c) American Mathematical Society 1972 
$n \geqq 0$, we have

$$
\int_{\gamma} \phi z^{n} d z=\int_{\gamma}(\phi \psi)\left(\bar{\psi} z^{n}\right) d z=\int_{\gamma}(\psi f)\left(\bar{\psi} z^{n}\right) d z=\int_{\gamma} f z^{n} d z=0
$$

which implies that $\phi \in H^{\infty}$. Similarly, $\bar{\phi} \in H^{\infty}$ implies $\phi$ is a constant.

COROLlary. If $\|\phi\|_{\infty} \leqq 1$ and if $\phi$ is not constant, then the map $u \mapsto u\left(T_{\phi}\right)$ from $H^{\infty}$ into $B\left(H^{2}\right)$ defined by

$$
u\left(T_{\phi}\right)=\lim _{r \rightarrow 1-0} \sum_{k=0}^{\infty} C_{k} r^{k} T_{\phi}^{k},
$$

where $u(\lambda)=\sum_{k=0}^{\infty} C_{k} \lambda^{k} \in H^{\infty}$ is a norm decreasing homomorphism of the algebra $H^{\infty}$ into $B\left(H^{2}\right)$.

Proof. Apply the above theorem and Theorem 2.1, Chapter III of [2].

\section{REFERENCES}

1. P. Halmos, A Hilbert space problem book, Van Nostrand, Princeton, N.J., 1967. MR 34 \#8178.

2. B. Sz.-Nagy and C. Foiaş, Analyse harmonique des opérateurs de l'espace de Hilbert, Masson, Paris; Akad. Kiadó, Budapest, 1967. MR 37 \#778.

Department of Mathematics, University of Michigan, Ann Arbor, Michigan 48104 